CAHIERS DE

NARRATOLOGIE

\section{Cahiers de Narratologie}

Analyse et théorie narratives

$13 \mid 2006$

Nouvelles approches de l'intertextualité

\title{
Pour une définition opérationnelle de la parodie littéraire: parcours critique et enjeux d'un corpus spécifique
}

\section{Yen-Mai Tran-Gervat}

\section{OpenEdition}

Journals

Édition électronique

URL : http://journals.openedition.org/narratologie/372

DOI : $10.4000 /$ narratologie.372

ISSN : 1765-307X

Éditeur

LIRCES

Référence électronique

Yen-Mai Tran-Gervat, « Pour une définition opérationnelle de la parodie littéraire: parcours critique et enjeux d'un corpus spécifique », Cahiers de Narratologie [En ligne], 13 | 2006, mis en ligne le 01

septembre 2006, consulté le 19 avril 2019. URL : http://journals.openedition.org/narratologie/372 ;

DOI : 10.4000/narratologie.372

Ce document a été généré automatiquement le 19 avril 2019.

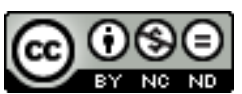

Cahiers de Narratologie - Analyse et théorie narratives est mis à disposition selon les termes de la licence Creative Commons Attribution - Pas d'Utilisation Commerciale - Pas de Modification 4.0 International. 


\title{
Pour une définition opérationnelle de la parodie littéraire: parcours critique et enjeux d'un corpus spécifique
}

\author{
Yen-Mai Tran-Gervat
}

1 Depuis la parution de Palimpsestes, de G. Genette $(1982)^{1}$, la critique française se méfie avec raison du mot «parodie », que la « vulgate » (Genette) a tendance à dissoudre dans l'intertextualité, la caricature ou la satire. Pourtant, une telle conscience du terme critique et de son bon usage ne parvient pas à dissiper totalement les effets de lecture spontanés qu'offrent certaines œuvres formant a priori un corpus parodique cohérent ${ }^{2}$.

2 C'est cette tension entre la rigueur théorique et terminologique d'une part et la prise en compte, d'autre part, de la réception de la parodie, ou peut-être plus précisément, du paro dique ${ }^{3}$ comme effet particulier de décalage comique du texte par rapport à une norme connue, qui sera au centre de cet article. Nous proposerons une définition opérationnelle de la parodie, suffisamment précise pour ne pas devenir un fourre-tout et en même temps suffisamment large pour permettre de rendre compte de la diversité des pratiques " parodiques » repérables dans les œuvres singulières.

3 «Ni la «minimaliser », ni la voir partout... », pour reprendre une expression de Daniel Sangsue $^{4}$, tel est le juste milieu que nous rechercherons dans cette définition de la parodie littéraire. Pour ce faire, nous devons nous positionner sur un "spectre" théorique de la parodie, que l'on peut résumer par les définitions suivantes, du sens le plus strict au sens le plus large :

- 1. «Détournement de texte à transformation minimale ( (G. Genette, Palimpsestes, p. 40) ;

«Transformation ludique d'un texte singulier» (p. 202).

- 2. «Transformation ludique, comique ou satirique d'un texte singulier» (D. Sangsue, p. 73).

- 3. «Refonctionnement comique d'un matériau linguistique ou artistique préformé » (M. Rose $^{5}$, p. 52).

- 4. Répétition avec une distance critique (par renversement ironique), qui insiste plus sur la différence que sur la ressemblance (L. Hutcheon ${ }^{6}$, p. 6). 
- 5. Dénudation comique d'un procédé littéraire mécanisé, permettant son renouvellement (définition reconstituée, Tomachevski ${ }^{7}$, p. 301).

- 6. Cas particulier de stylisation d'un discours autre, qu'il soit littéraire ou social. En ce sens, la parodie est un cas particulier du dialogisme et du plurilinguisme propres au discours romanesque (Définition reconstituée, Bakhtine ${ }^{8}$ ).

\section{«fonctionnelle» des "pratiques hypertextuelles», dont la parodie n'est qu'un cas} particulier. « régime» (l'intention ou l'effet de chaque pratique, qui peut être ludique, satirique ou sé rieuse) et la "relation" entre l'hypertexte et l'hypotexte, qui consiste soit en une transformation, soit en une imitation. La parodie est, dans ces termes, une " transformation textuelle à fonction ludique ».

La fonction ludique est introduite par G. Genette pour rendre compte des pratiques qui visent « une sorte de pur amusement ou exercice distractif, sans intention agressive et moqueuse $"^{9}$ vis-à-vis du texte imité ou transformé. L'appartenance de la parodie à ce ré gime la distingue selon G. Genette du travestissement (satirique) et de la transposition (sérieuse), avec lesquels elle partage une relation transformationnelle vis-à-vis de l'hypo texte; cette relation la différencie radicalement du pastiche, avec lequel elle partage pour tant le régime ludique : la parodie transforme, quand le pastiche imite.

7 Seules deux pratiques bénéficient chez G. Genette de ce qu'on pourrait appeler «l'Appellation Parodie d'Origine Structurale Contrôlée ».

8 G. Genette isole d'abord la "parodie stricte » ou « détournement de texte à transforma tion minimale ", dont l'exemple canonique est Le Chapelain décoiffé de Boileau et Racine ${ }^{10}$. La parodie apparaît comme une figure micro-structurale, proche de la citation détournée, dont les jeux oulipiens fournissent des exemples très convaincants.

9 Daniel Sangsue, dans son essai sur la parodie, propose d'élargir la définition de G. Genette à d'autres régimes que le seul régime ludique : «transformation ludique, comique ou satirique d'un texte singulier ». Ce faisant, il conserve la formulation de G. Genette impliquant strictement une relation de transformation et un hypotexte « singulier », mais il l'élargit légitimement au régime satirique, c'est-à-dire au «travestissement bur lesque ». Notre propre corpus théorique ${ }^{11}$ et romanesque du XVIII ${ }^{e}$ siècle a confirmé la nécessité de cette extension, car il apparaît dans ces textes que l'effet parodique repose davantage sur le contraste comique créé par la réécriture transformatrice que sur l'intention (moqueuse ou non) de son auteur. L'importance de ce contraste est d'ailleurs telle pour les parodistes qu'elle autorise d'autre part à considérer comme "parodique " au XVIII ${ }^{e}$ siècle le contraste héroï-comique, pourtant fondé sur une structure imitative.

Pour la deuxième espèce de parodie (dont D. Sangsue ne rend pas compte), G. Genette s'ap puie sur une appellation historique: celle des "parodies dramatiques» telles qu'elles étaient pratiquées sur les scènes des Italiens ou de la Foire au XVIII ${ }^{\text {e }}$ siècle. G. Genette ap pelle ce procédé " parodie mixte ", " eu égard à [sa] structure complexe et indécise », qui " hésite entre les diverses possibilités de la parodie, du burlesque, et de l'héroï-comique » ${ }^{12}$. La parodie mixte consiste en effet à donner aux personnages du texte parodié des doubles de condition inférieure, parlant leur propre langage : il ne s'agit donc pas du burlesque traditionnel (chez Scarron, les héros de Virgile parlant comme des cro 
cheteurs), mais d'une forme originale de parodie fondée sur une « homologie » entre la parodie et sa cible (« hypertexte » et « hypotexte »), « mais l'étage en-dessous ».

11 La parodie mixte ${ }^{13}$ nous offre l'occasion de fonder une définition élargie de la parodie sans forcément contredire la rigueur terminologique de Palimpsestes. Elle permet en effet d'ouvrir une brèche dans le système de classification de G. Genette, en particulier dans son refus de la «parodie de genre $»^{14}$ : la parodie stricte transforme un texte singulier, de préférence bref, mais la parodie mixte vise des œuvres étendues, représentatives d'un certain style et peut-être même d'un certain genre (Agnès de Chaillot, parodie de la tragé die d'Inès de Castro ; Le Télémaque travesti, parodie du roman pédagogique de Fénelon).

G. Genette se trouve confronté au même problème lorsqu'il prend en considération Don Quichotte et ses successeurs (Le Berger extravagant de Sorel, les romans de jeunesse de Marivaux), créateurs d'un genre que, faute d'un terme plus précis et afin d'éviter le mot "parodie ", il appelle après Sorel l'« antiroman", et dont l'hypotexte " est en fait un hypo-genre $»^{15}$. G. Genette ne saurait nier les liens de l'antiroman avec la parodie, dont il s'efforce pourtant de le distinguer sans ambiguïté :

Comme dans la parodie, et mieux encore dans la parodie mixte, il arrive aux héros vulgaires de l'antiroman des aventures analogues à celles des héros de genres nobles. Mais dans les parodies, il s'agit d'une analogie réelle, inconsciente, et purement diégétique. Dans l'antiroman au contraire, l'analogie est métadiégétique, entièrement située dans l'esprit et le discours du héros, qui la perçoit non seulement comme une analogie mais comme une identité, et dénoncée (et conçue) comme illusoire par l'auteur et par le public ${ }^{16}$.

13 Pour G. Genette, l'antiroman « s'apparente » donc à la parodie et à l'héroï-comique : mis à part la volonté déterminée de définir la parodie autrement que « la vulgate », on voit mal ce qui empêche de voir dans l'antiroman un cas particulier de parodie mixte, initiée par la folie romanesque... Le Télémaque travesti, donné comme exemple de parodie mixte un peu plus haut, devient d'ailleurs dans ce chapitre un antiroman à hypotexte singulatif, ou un "pseudo-Télémaque fort abouti »...

Comme l'annonce lui-même G. Genette, l'analyse des œuvres met donc à l'épreuve les catégories qu'il a soigneusement définies au début de son essai : il préfère alors morceler ces catégories en distinctions de plus en plus fines, chaque texte formant une catégorie différente qu'il ne faut surtout pas confondre avec la parodie...

Il nous semble pour notre part que l'analogie hypertextuelle illusoire qu'introduit la folie romanesque dans "l'antiroman " peut être sans erreur conceptuelle considérée comme une "transformation textuelle à fonction ludique ", si l'on prend en compte d'une part le choix littéraire (transformation, fonction ludique) de l'auteur dans la création d'un tel roman, d'autre part le phénomène de réception commun à l'antiroman et à la parodie, que G. Genette signale sans s'y attarder. De plus, la dimension «métadiégétique » qui fonde chez Genette la différence entre antiroman et parodie nous paraît largement présente dans le procédé parodique, même entendu au sens le plus strict, le décalage comique, ou la «transformation ludique " repérée par le lecteur étant une rupture de l'illusion fictionnelle voulue et exhibée par le parodiste, que la relation hypertextuelle soit « diégétique » ou " métadiégétique ».

La dimension métatextuelle de la parodie a été récemment mise en avant par les théoriciens, notamment dans les pays anglo-saxons, afin de rendre compte des pratiques parodiques modernistes (Joyce) et "post-modernes » (J. Fowles, J. Barth), en particulier dans le roman. Or ces critiques ne cachent pas leur filiation critique avec les formalistes 
russes et Bakhtine, dont la conception de la parodie, à l'autre bout de notre "spectre", participe de manière décisive et globale à une théorie évolutionniste de la littérature. Là, la parodie est tout entière définie par sa fonction, aux antipodes de la démarche structurale de G. Genette.

Lorsque les formalistes russes se proposent d'élaborer une théorie de la littérature, ils ont en effet recours à une conception très large de la parodie, souvent confondue avec le pastiche: dans les textes des formalistes, ces deux mots sont pratiquement synonymes pour désigner la citation « transcontextualisée » (L. Hutcheon) de textes ou procédés litté raires.

La relation d'imitation ou de transformation, centrale chez G. Genette, est ici subshumée par la fonction transformatrice et rénovatrice de la parodie-pastiche ; que celle-ci imite ou transforme le texte parodié, sa fonction est toujours de mettre à nu des procédés " mécanisés », caractères usés d'un genre ou d'un style donné, et de les réinvestir dans un nouveau contexte, où leur reconnaissance par le lecteur s'accompagne d'un effet co mique. Dans son article "Thématique ", Tomachevski fait ainsi intervenir la parodiepastiche dans la « dénudation » des « procédés canoniques perceptibles»:

Parmi les œuvres qui dénudent leurs procédés, il faut isoler celles qui révèlent un procédé étranger à l'œuvre, soit traditionnel, soit propre à un autre écrivain. S'il résulte un effet comique de la dénudation d'un procédé littéraire, nous avons affaire à un pastiche. Les fonctions du pastiche sont multiples. On cherche habituellement à ridiculiser l'école littéraire opposée, à détruire son système créateur, à le « dévoiler».

[...]

Pourquoi la dénudation du procédé existe-t-elle? Le procédé perceptible ne se justifie esthétiquement que lorsqu'il est volontairement rendu perceptible. Un procédé perceptible masqué par l'auteur produit une impression comique (aux dépens de l'œuvre). Prévenant cette impression, l'auteur révèle le procédé.

Ainsi les procédés naissent, vivent, vieillissent et meurent. Au fur et à mesure de leur application, ils deviennent mécaniques, ils perdent leur fonction, ils cessent d'être actifs. Pour combattre la mécanisation du procédé, on le renouvelle grâce à une nouvelle fonction ou à un sens nouveau. Le renouvellement du procédé est analogue à la citation d'un auteur ancien dans un contexte nouveau et avec une signification nouvelle ${ }^{17}$.

Dans ces dernières lignes, Tomachevski définit explicitement la «dénudation du procédé ", qui a tout de la parodie de genre ou de style, comme l'analogue de ce que G. Genette pourrait appeler la parodie stricte: leur point de rencontre est la fonction rénovatrice de la répétition ou de la citation volontairement détournée.

On veillera cependant à ne pas confondre trop hâtivement le mouvement parodique et l'évolution littéraire en général, telle que la conçoivent les formalistes : la parodie n'est qu'un procédé parmi d'autres du renouvellement «naturel» des styles et des genres (intertextualité) et même, de la mise à nu des procédés (métatextualité); ce n'est donc pas une pratique universelle, omniprésente et polymorphe, mais bien un choix stylistique et littéraire précis, dont il faut prendre en considération les différentes formes, fonctions et conditions d'utilisation, dans les œuvres individuelles où elle se manifeste ouvertement.

21 M. Bakhtine précise et étend à la fois le champ de la parodie littéraire telle que la concevaient les formalistes, lorsqu'il la met au centre de sa réflexion sur le discours du $\operatorname{roman}^{18}$, mais aussi lorsqu'il définit le principe de la littérature carnavalesque, de Rabelais à Dostoïevski. 
bien compte de cette réflexivité créatrice propre au roman et plus particulièrement encore, à la parodie :

On peut dire sans détours que la prose romanesque européenne naît et s'élabore

dans un processus de traduction libre (transformatrice) des œuvres d'autrui ${ }^{24}$.

Pourtant, L. Hutcheon insiste avec Bakhtine sur l'ambivalence de la force de subversion que représente la parodie, de manière analogue au «rire carnavalesque » qu'il définit dans ses textes sur Rabelais et Dostoïevski. L'expression "authorized transgression " utilisée par L. Hutcheon résume le paradoxe du carnaval comme celui de la parodie: Bakhtine décrit le carnaval médiéval comme une subversion temporaire de l'ordre social et ecclésiastique, ce qui suppose que ces fêtes sont tolérées, légalisées par les autorités ellesmêmes, qui s'effacent pendant un temps qui ne dépend que d'elles ${ }^{25}$. Or une telle ca ractéristique est partagée par le discours ou le texte parodique :

Ce paradoxe d'une subversion légalisée bien que non-officielle caractérise tout discours parodique, dans la mesure où la parodie suppose, comme condition de son

Cahiers de Narratologie, 13 | 2006 
existence même, une certaine institutionalisation esthétique qui permette la reconnaissance de formes et de conventions stables et familières ${ }^{26}$.

L'analogie entre le carnaval et la parodie permet à L. Hutcheon de souligner que la parodie, en même temps qu'elle déforme et subvertit sa cible, la conserve et la respecte, en l'intégrant de manière reconnaissable dans sa propre forme: la "stylisation paro dique " est un outil critique subtil et complexe, possédant à la fois des tendances « normatives et conservatrices » et des tendances « provocatrices et révolutionnaires » ${ }^{27}$.

Nous avons exposé les théories qui se trouvent aux deux pôles extrêmes du "spectre » définitionnel de la parodie, en essayant de souligner à chaque fois les voies d'interpré tation qu'elles offrent pour l'étude concrète des textes.

Cette exigence pragmatique trouve un écho dans la «troisième voie » explorée par deux théoriciennes anglo-saxonnes : c'est d'abord Linda Hutcheon, dont la Theory of Parody (1986), malgré sa perspective « post-moderne » appuyée, semble utile à bien des égards y compris pour des textes antérieurs au $\mathrm{XX}^{\mathrm{e}}$ siècle, notamment pour sa formulation du « paradoxe de la parodie ».

32 Mais cette idée est également formulée très clairement, et s'appuie sur une démarche plus spécifiquement historique et critique dans Parody: Ancient, Modern, and Post-Modern (1993), de l'universitaire australienne et britannique Margaret Rose, dont les analyses et les propositions recoupent largement nos propres intuitions ${ }^{28}$.

Toutes deux ont retenu les distinctions de G. Genette mais, en s'appuyant notamment sur Aristote et Bakhtine, cherchent à étendre le champ de la parodie au-delà de ces caté gories, sans pour autant retomber dans l'indistinction. L. Hutcheon, dont le sujet principal reste l'art contemporain ${ }^{29}$, oriente surtout cet élargissement vers la " parodie sérieuse » suggérée par le « régime sérieux » de G. Genette. M. Rose est quant à elle plus proche de notre propre démarche lorsqu'elle cherche à montrer que la notion de parodie comique ne se limite pas à la "parodie stricte » (specific parody), mais peut également s'appliquer à des œuvres entières, surtout des romans, dans le cadre de ce qu'elle appelle la « parodie générale » (general parody) :

Dans toutes ces variantes «strictes » ou "générales », la parodie peut être définie en termes généraux comme le refonctionnement comique d'un matériau linguistique ou artistique préformés ${ }^{30}$.

4 De G. Genette, on ne peut que retenir la mise en garde contre la confusion de la parodie avec des notions voisines, et la prise en compte du « régime » et de la "relation » à l'œuvre dans la pratique parodique : Palimpsestes aura mis l'accent sur les rapports, de formes et d'intentions diverses, entre l'hypertexte et l'hypotexte.

Nous distinguons donc la parodie d'abord de la satire, dont la cible est largement extralittéraire (une personnalité, un fait de société) alors que celle de la parodie littéraire est interne à la littérature (canons d'un genre ou d'un style " usé », une œuvre singulière et sa conception de la fiction) ; cette distinction n'empêche pas de concevoir une parodie à visée satirique, lorsqu'un détournement de texte (ludique) sert à se moquer d'un sujet non littéraire (c'est souvent le cas de l'héroï-comique). Quant à ce que certains appellent la « satire littéraire ", c'est-à-dire une transformation textuelle qui entend se moquer du texte même qu'elle cite (Shamela de Fielding), elle pourra être considérée comme une forme de parodie (stricte ou mixte), avec une fonction critique ou polémique qui ne semble pas exclure la dimension ludique. 

imite plus qu'il ne transforme, alors que la parodie se signale par l'écart manifeste, souvent source de comique, qu'elle établit avec sa cible-modèle. Nous retenons avec G. Genette que le trait distinctif de la parodie est la prédominance de la transformation par rapport à l'imitation, dans la manière de réécrire son matériau littéraire de départ : un texte chez G. Genette, que nous étendons pour notre part à un style ou à un genre.

Nous proposons donc de délimiter la notion de parodie en insistant sur les aspects suivants :

- la tension dynamique entre l'imitation et la transformation de l'hypotexte par la parodie, tension manifeste grâce à la co-présence explicite ou implicite dans la parodie de son hypotexte ; la relation de transformation nous semble en effet essentielle dans la parodie, mais elle n'y est perceptible que sur fond d'imitation. Cette double nature de la parodie (imitation, transformation) constitue son ambivalence propre, analogue à celle du carnavalesque bakhtinien.

- L'appartenance de la parodie au « régime ludique », qui nous semble inclure la notion de co mique, la mise à distance ironique et peut-être aussi l'intention satirique (à l'égard d'un texte littéraire identifiable) ;

- la mise en place d'un contraste comique pour le lecteur entre ce qu'il reconnaît et ce que la parodie détourne, « refonctionne »; en cela, la cible de la parodie ne se limite pas forcément à un texte singulier mais, sur une échelle qui peut varier du mot d'auteur au procédé générique, sera reconnaissable par le lecteur comme un système littéraire identifiable par certaines caractéristiques saillantes, que la parodie reprend et transforme.

- la dimension de création et de rénovation stylistique et générique du recours à la parodie, largement réflexive.

- Ces conditions étant posées, nous entendons donc par parodie littéraire la réecriture ludique d'un système littéraire reconnaissable (texte, style, stéréotype, norme générique...), exhibé et trans formé de manière à produire un contraste comique, avec une distance ironique ou critique.

Cette définition « opérationnelle » de la parodie est destinée à servir d'appui et de gardefou à toute étude de textes considérés comme parodiques : l'effet de lecture immédiat doit pouvoir être vérifié par la confrontation avec ces critères de reconnaissance, puis nuancé par une typologie des pratiques parodiques et de leurs fonctions dans les textes ${ }^{31}$.

\section{NOTES}

1. G. Genette, Palimpsestes. La littérature au second degré, Paris, Seuil, coll. « Points », 1982, $565 \mathrm{p}$.

2. Tel celui que nous avons étudié par ailleurs comme relevant du « roman parodique au XVIIIe siècle en Angleterre et en France, de Marivaux à Jane Austen ", thèse de doctorat en littérature comparée, Paris IV (co-direction d'Y. Chevrel et J. Dagen), soutenue le 14 décembre 2000.

3. En privilégiant l'adjectif, on réintroduit un élargissement fonctionnel du sens, analogue à celui de « comique » par rapport à « comédie ». 
4. D. Sangsue, La Parodie, Paris, Hachette Supérieur, coll. « Contours littéraires », 1994, 106 p. La citation se trouve à la p. 5. L'auteur cherche lui-même à formuler une définition « opérationnelle » de la parodie.

5. M. Rose, Parody: Ancient, Modern and Post-Modern, Cambridge University Press, «Literature, Culture, Theory » $n^{\circ}$ 5, 1993, 316 p.

6. L. Hutcheon, A Theory of Parody, London, Methuen, 1986, 143 p.

7. B. Tomachevski, « Thématique », 1925, dans Théorie de la littérature, textes des formalistes russes réunis, présentés et traduits par T. Todorov, Paris, Seuil, 1965. 8. M. Bakhtine, notamment dans « Du discours romanesque ", deuxième étude in Esthétique et théorie du roman, Paris, Gallimard, «Tel », 1978, pp. 83-233.

9. Ibid., p. 43.

10. Exemple : «O rage, ô désespoir! O perruque ma mie N'as-tu donc tant duré que pour tant d'infâmie? » Cité par G. Genette, ibid., p. 30.

11. V. par exemple Dumarsais, Des Tropes, ou des différents sens dans lesquels on peut prendre un même mot dans une même langue (1ère édition, 1730), Paris, David, 1757, 310 p. Livre III, chap. 10 : «Du sens adapté », p. 273-281; Marmontel, Eléments de littérature, Euvres complètes, T. V à X, Paris, Née de la Rochelle, 1787, articles « Burlesque » et « Parodie », parus précédemment dans l'Encyclopédie ; L. Riccoboni, Observations sur la parodie, quatrième et dernier livre des Observations sur la comédie et sur le génie de Molière, 1736, édition en fac-simile par Arnaldo Forni Editore, 1978, pp. 275-348.

12. Cette hésitation constatée du point de vue structural par Genette confirme la nécessité, d'un point de vue fonctionnel, d'étendre la notion de parodie au burlesque et à l'héroï-comique notamment.

13. Que G. Genette décrit et étudie notamment à partir du Télémaque travesti de Marivaux (1714), op. cit., p. 194-198.

14. Qui relève selon lui du pastiche, car on ne peut qu'imiter un genre ou un style (général) et transformer un texte (singulier). V.ibid., p. 111 : « La notion si répandue de « parodie de genre » est une pure chimère, sauf à y entendre, explicitement ou implicitement, parodie au sens d'imitation satirique ».

15. Ibid., p. 209.

16. Ibid., p. 206-207.

17. B. Tomachevski, « Thématique », 1925, dans Théorie de la littérature, p. 301.

18. Nous nous référons essentiellement aux articles réunis dans Esthétique et théorie du roman, Paris, Gallimard, Tel, 1978.

19. V. Esthétique et théorie du roman, Deuxième étude : « Du discours romanesque », p. 86-233.

20. Ibid., p. 180.

21. Ibid., p. 122-123.

22. M. Aucouturier : préface de M. Bakhtine, Esthétique et théorie du roman, Gallimard-Tel, p. 18 .

23. L. Hutcheon, op. cit., Chap. 4 : « The Paradox of Parody », p. 69-83.

24. M. Bakhtine, op. cit., p. 193. Les italiques sont de l'auteur.

25. Cf. L. Hutcheon, op. cit., p. 74 : « La reconnaissance du monde inversé suppose toujours une connaissance du monde dont réalise l'inversion et en quelque sorte l'intégration. » (notre traduction)

26. Ibid., p. 74-75, nous traduisons.

27. L. Hutcheon, op. cit., p. 76. 
28. V. en particulier son premier chapitre, à la fois très documenté et très clairement argumenté : « Ways of defining parody », p. 5-53.

29. Le sous-titre de son ouvrage est : « The Teachings of XXth Century Art Forms ».

30. M. Rose, op. cit., p. 52 (notre traduction).

31. Sans négliger bien entendu le sens que prend le recours à la parodie en fonction des données historiques et théoriques propres aux ères culturelles étudiées, à la période choisie et au genre littéraire considéré.

\section{RÉSUMÉS}

L'article propose une définition opérationnelle de la parodie, pouvant servir d'outil de reconnaissance, d'analyse et d'interprétation de la parodie littéraire dans les textes qui la mettent en œuvre. Cette définition tient compte des enseignements des principales théories de la parodie au XXe siècle (G. Genette, les formalistes russes, Bakhtine, et les théories anglosaxonnes), mais aussi de l'effet parodique immédiat d'un corpus spécifique, où la rigueur théorique doit tenir compte de la cohérence propre du texte étudié.

\section{AUTEUR}

\section{YEN-MAI TRAN-GERVAT}

Université de Poitiers 